\title{
Photoenhanced injection currents in organic solar cells
}

\author{
Holger Frohne, ${ }^{\text {a) }}$ Christopher R. McNeill, and Paul C. Dastoor \\ School of Mathematical and Physical Sciences, University of Newcastle, Callaghan, NSW 2308, Australia
}

(Received 12 April 2004; accepted 10 June 2004)

\begin{abstract}
Bulk heterojunction organic solar cells with the general structure: indium tin oxide/ polyethylenethioxythiophene (PEDOT)/bulk-heterojunction layer/Al with built-in fields between -0.1 and $1 \mathrm{~V}$ have been fabricated by electrochemically doping the PEDOT electrode. The active bulk-heterojunction layer consisted of a blend of MDMO-PPV (poly(2-methoxy-5(3', 7'-dimethyl)octyloxy-1,4-phenylenevinylene)) and PCBM $\left((6,6)\right.$-phenyl- $\mathrm{C}_{61}$-butyric-acid methyl ester)). Measurements of the current-voltage curves with varying light intensities for these devices reveal the presence of light-dependent currents in addition to those from the photogenerated charge carriers. Equivalent circuit modeling indicates that these light-dependent currents most likely originate from photoenhanced injection at the electrodes. (C) 2004 American Institute of Physics.

[DOI: 10.1063/1.1779354]
\end{abstract}

Organic solar cells (OSCs) based on polymeric rather than conventional inorganic semiconducting materials offer the possibility of inexpensively producing large area photovoltaic units. ${ }^{1}$ These materials can be readily deposited on mechanically flexible substrates and thus a variety of device geometries are possible.

A common procedure for characterizing organic solar cells is the measurement of current-voltage $(I-V)$ curves under illumination and allows the short circuit current, the open circuit voltage $\left(V_{\mathrm{oc}}\right)$, the fill factor, and subsequently the power conversion efficiency to be determined. The overall electrical field caused by both the built-in field $\left(V_{\mathrm{bi}}\right)$ and the applied voltage $\left(V_{\text {ext }}\right)$ drives photogenerated charge carriers toward the electrodes. In addition, currents originating from charge injection at the electrodes play an important role for photovoltaic device performance, although they are typically neglected in most descriptions of OSC characteristics.

We investigated bulk-heterojunction $\mathrm{OSCs}^{1}$ with the general structure: indium tin oxide (ITO)/ polyethylenethioxythiophene (PEDOT)/bulk-heterojunction layer/Al. The bulk-heterojunction layer consisted of a blend of MDMO-PPV (poly(2-methoxy-5-(3', $7^{\prime}$ dimethyl)octyloxy-1,4-phenylenevinylene)) and PCBM $\left((6,6)\right.$-phenyl- $\mathrm{C}_{61}$-butyric-acid methyl ester $\left.)\right)$. For these devices a desired $V_{\mathrm{bi}}$ can be adjusted in a range between -0.1 and $1 \mathrm{~V}$ by electrochemical doping of the PEDOT layer at the respective equilibrium potential $E_{\text {eq }}$. Details for preparing the devices are described elsewhere. ${ }^{2}$ For an ideal device under sufficient illumination the $I-V$ curves are expected to cross the voltage axis exactly at the value given by their flatband condition (i.e., $V_{\mathrm{oc}}$ ). However, lowering the work function difference $\left(\Delta \phi_{w}\right)$ between the electrodes makes $V_{\mathrm{oc}}$ ill-defined. ${ }^{3}$ For example, $I-V$ curves of an OSC with a small inverted built-in field $\left(V_{\mathrm{bi}} \approx-0.12 \mathrm{~V}\right)$ at different incident light intensities [Fig. 1(a)] intersect below the axis exactly at a single point. For a typical device with a highly doped PEDOT layer $\left(V_{\mathrm{bi}} \approx 1 \mathrm{~V}\right)$ the curves intersect just above the axis [Fig. 1(b)].

Considering the equivalent circuit shown in Fig. 2(a) it is straightforward to write down the total current $I$, in terms of

\footnotetext{
${ }^{a)}$ Electronic mail: holger.frohne@newcastle.edu.au
}

the diode current $I_{\text {diode }}$, the photogenerated current $I_{\mathrm{ph}}$, and the shunt current $I_{\text {sh }}$, which originates from the shunt resistance $R_{\mathrm{sh}}$ and the series resistance $R_{s}$ :

$$
I=I_{\mathrm{diode}}+I_{\mathrm{sh}}-I_{\mathrm{ph}}=I_{0}\left(e^{(q / n k T)\left(U-I R_{s}\right)}-1\right)+\frac{U-I R_{S}}{R_{\mathrm{sh}}}-I_{\mathrm{ph}}
$$

$I_{\mathrm{ph}}$ results from the photogenerated charge carriers produced in the OSC under illumination. $I_{\text {diode }}$ is given by the Shockley equation, in which $I_{0}$ is the diode saturation current, $q$ the elementary charge, $n$ the diode ideality factor, $k$ the Boltzmann constant, and $T$ the temperature.
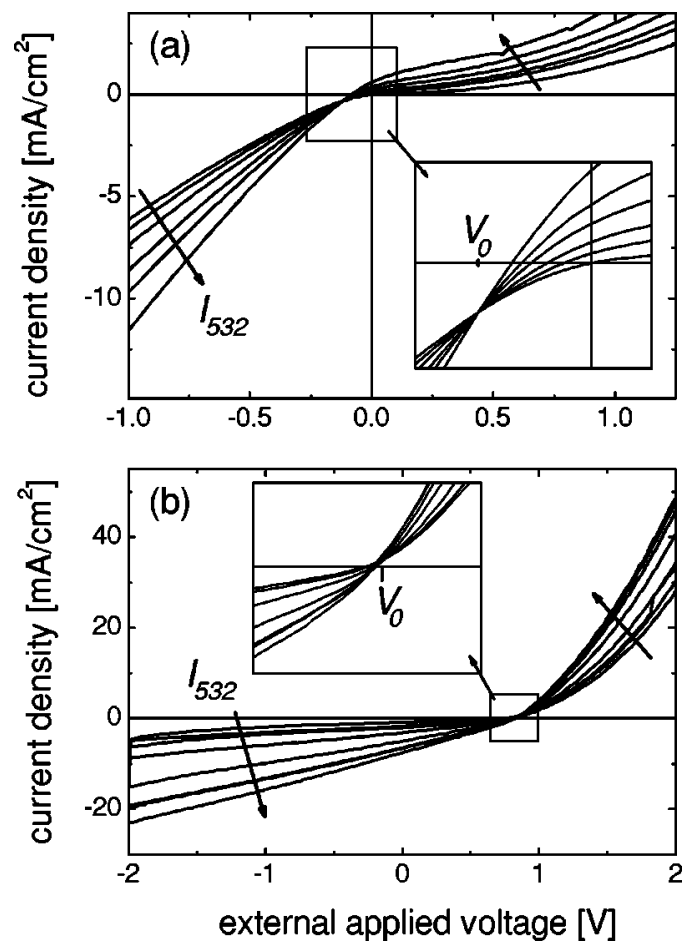

FIG. 1. $I-V$ curves of OSCs with an active area of $6.3 \mathrm{~mm}^{2}$ illuminated with a $532 \mathrm{~nm}$ laser at incident intensities $I_{532}$ of $0,45,70,120,200$, and $330 \mathrm{~mW} / \mathrm{cm}^{2}$ (sequence is determined by the arrows). (a) Inverted lowbuilt-in-field OSC $\left(V_{0}=-0.12 \mathrm{~V}\right), R_{\mathrm{sh}}=6 \mathrm{k} \Omega$. (b) normal OSC $\left(V_{0}=0.8 \mathrm{~V}\right)$, $R_{\text {sh }}=75 \mathrm{k} \Omega$. 

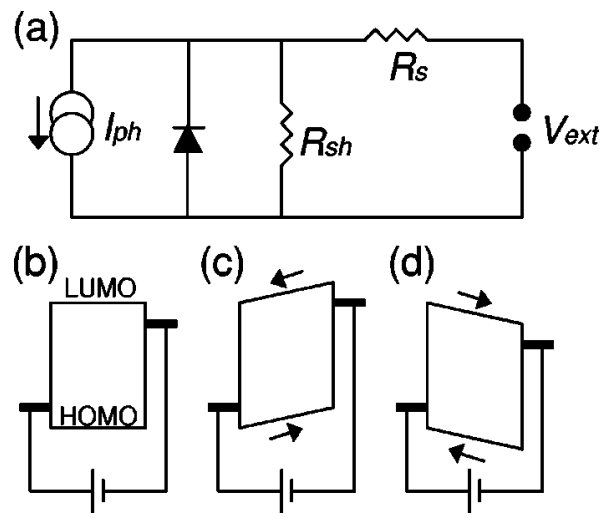

FIG. 2. (a) Equivalent circuit for a single junction solar cell. The circuit exhibits a photocurrent $I_{\mathrm{ph}}$, a diode current $I_{\text {diode }}$, and a shunt current $I_{\mathrm{sh}}$. (b)-(d) Simplified energy diagrams of an illuminated OSC at three external voltages $V_{\text {ext }}$ : (b) OSC driven at exactly $V_{\text {ext }}=V_{\text {bi }}$, (c) OSC driven at $V_{\text {ext }}$ $=V_{\mathrm{bi}}+\Delta V$, (d) OSC driven at $V_{\mathrm{ext}}=V_{\mathrm{bi}}-\Delta V$. The tilt for photogenerated charge carriers in (c) and (d) is identical. Therefore, photogenerated currents are considered to be symmetrical around $V_{\mathrm{bi}}$.

We can now explain the shift in the intersection of the $I-V$ curves at different incident light intensities by a finite value of $R_{\mathrm{sh}}$. $R_{\mathrm{sh}}$ shifts this intersection from a generally assumed $V_{\mathrm{oc}}$ at $I=0$ to a fixed current value at $V_{0}$, at which voltage the net photocurrent (the total measured current at a certain incident light intensity minus the total measured dark current) is zero. ${ }^{4}$ From the data shown in Fig. 1, values for $R_{\text {sh }}$ are calculated to be 8 and $75 \mathrm{k} \Omega$ for the inverted and high built-in field devices, respectively.

$I_{\mathrm{ph}}$ is determined by the photon intensity and the internal electrical field during the measurement. As illustrated by the simplified energy diagram for an OSC [Figs. 2(b)-2(d)], when $V_{\text {ext }}=V_{\mathrm{bi}}$ the device is forced back to its flatband condition and $I_{\mathrm{ph}}$ is zero. Therefore, for a given incident photon flux, the $I_{\mathrm{ph}}$ produced by an applied voltage $V_{\mathrm{ext}}=V_{\mathrm{bi}}+\Delta V$ [Fig. 2(c)] must be equal and opposite to the $I_{\mathrm{ph}}$ produced by an applied voltage $V_{\text {ext }}=V_{\mathrm{bi}}-\Delta V$ [Fig. 2(d)], since the magnitude of the band gradients is the same in both cases. This symmetry argument can be used to eliminate $I_{\mathrm{ph}}$ from the total measured current by a pairwise summation of the current at every value of $V_{\mathrm{ext}}=V_{\mathrm{bi}} \pm \Delta V_{j}$ for all $\Delta V_{j}$ and thus:

$$
I_{\mathrm{sum}}\left(V_{\mathrm{bi}}+\Delta V_{j}\right)=\sum_{ \pm}\left\{I\left(V_{\mathrm{bi}} \pm \Delta V_{j}\right)\right\}=I_{\mathrm{diode}}^{\mathrm{sum}}+I_{\mathrm{sh}}^{\mathrm{sum}},
$$

since the pairwise photocurrent contributions in $I_{\mathrm{ph}}^{\mathrm{sum}}$ cancel. Figures 3(a) and 3(b) show the calculated values of $I_{\text {sum }}$ for the devices mentioned earlier. Both devices exhibit nonlinear curves that vary with the incident light intensity. From Eq. (2), the light intensity dependence of the curves can only originate from either a light-dependent $I_{\text {diode }}^{\text {sum }}$ or $I_{\mathrm{sh}}^{\text {sum }}$ term.

The conductivity of PEDOT can be altered by photodoping $^{5}$ and thus may provide a mechanism for a lightdependent $I_{\text {sh }}^{\text {sum }}$. However, from Eq. (1), any light-dependent changes in $R_{\mathrm{sh}}$ or $R_{s}$ (and hence $I_{\mathrm{sh}}$ ) would change the intersection point of the $I-V$ curve with the voltage axis and thus prevent the $I-V$ curves from crossing in a single data point. Hence, it can be concluded that $I_{\text {diode }}^{\text {sum }}$ has to be light intensity dependent rather than $R_{s}$ or $R_{\mathrm{sh}}$.

Figures 3(a) and 3(b) illustrate that, with increasing light intensity, $I_{\text {sum }}$ becomes more positive for positively doped devices and more negative for negatively doped devices. Therefore, charge injection for $|V|>\left|V_{\mathrm{bi}}\right|$ (forward mode)

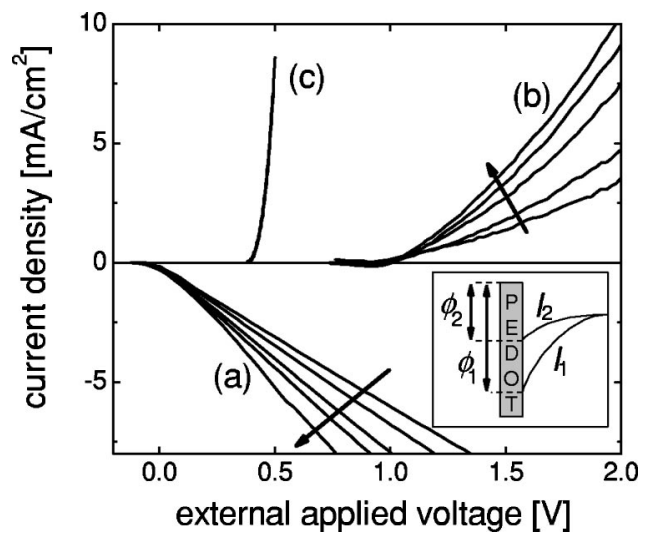

FIG. 3. (a)-(c) Results of pairwise summation of the total current around the flatband condition from measurements at different incident intensities (sequence is determined by the arrows: $45,70,120,200$, and $330 \mathrm{~mW} / \mathrm{cm}^{2}$ ) of $532 \mathrm{~nm}$ laser light. (a) OSCs at $V_{0}$ 's of $-0.12 \mathrm{~V}$ (curves below $x$ axis); (b) at $0.75 \mathrm{~V}$ (curves above $x$ axis); (c) same calculation for measurements of an inorganic solar cell (UDT-PIN 10D 9724-1). Inset: Photoenhanced injection current in OSCs: For a normal device the PEDOT's work function lies above the MDMO-PPV's HOMO energy level and thus at the junction band bending occurs to form a Schottky energy barrier. The Schottky barrier height $\left(\phi_{2}<\phi_{1}\right)$ changes with incident light intensity $\left(I_{2}>I_{1}\right)$.

must be light dependent and increases with increasing light intensity. Moreover, the proportion of the current generated via this photoenhanced charge injection is significant. Comparing the total photogenerated current (Fig. 1) with the photoinjected current indicates that photoenhanced injection accounts for $26 \%$ of the total current at $V_{\text {ext }}=2 V_{\text {bi }}$.

According to the energy level diagram displayed by the inset in Fig. 3, the (positively doped) PEDOT's work function lies above the MDMO-PPV's highest occupied molecular orbital (HOMO) level and thus at the junction band bending occurs to form a Schottky barrier. This barrier must be overcome if holes are to be injected into the device from the PEDOT electrode and hence we hypothesize that it is the Schottky barrier height that changes with incident light intensity. Photoexcitation of charge carriers from a semitransparent metal in conventional Schottky barrier devices is well-established and forms the basis of fast infrared detectors. ${ }^{6}$ More recently, near-infrared to visible upconversion has been shown to take place in conventional Schottky diodes $^{7}$ and ballistic transport of photoexcited electrons across the Schottky barrier in a Grätzel-type device has been demonstrated. ${ }^{8}$ Direct photoexcitation of excess carriers from the PEDOT into the polymer semiconductor would alter the space-charge distribution at the PEDOT/MDMOPPV:PCBM interface. Band bending in a $p$-type semiconducting polymer arises from a negatively polarized spacecharge region at the interface, ${ }^{9}$ which will be reduced by the presence of excess photo-injected holes thus lowering the Schottky barrier to injection as shown in the inset in Fig. 3. This proposed mechanism is further supported by recent measurements of the transient response of the modulated photocurrent in OSCs, which indicates that excess photogenerated carriers can alter the space-charge distribution at the Schottky barrier. ${ }^{10}$

It is interesting to compare the response of organic to conventional silicon devices under similar illumination conditions. Figure 3(c) shows the result of applying Eq. (2) to the $I-V$ curves measured for a silicon photodetector (UDTPIN 10D 9724-1). These curves exhibit the expected re- 
sponse with no intensity dependence for the variation of $I_{\text {sum }}$, indicating that the overall current is dominated by $I_{\mathrm{ph}}$.

OSCs differ structurally from conventional devices and consist of a compliant network of blended MDMO-PPV and PCBM forming a so-called bulk heterojunction structure ${ }^{1}$ rather than a rigid crystalline periodic lattice. Indeed, the degree of phase segregation that occurs in these blended systems is currently unclear ${ }^{11}$ and it is possible that segregation effects play a role in determining the magnitude of the proposed mechanism. Previous work has reported phase segregation in polyfluorene polymer blends, leading to a thin polymer rich interfacial layer. ${ }^{12}$ However, contradictory measurements of the Rutherford backscattering spectrum for an iodinated $\mathrm{C}_{60}$ derivative (I-PCBM) found that the PCBM is most likely distributed homogenously in the entire film. ${ }^{13}$ Ultimately, however, the presence of a phase segregated layer would simply alter the hole mobility and hence spacecharge distribution at the PEDOT interface without providing an alternative mechanism for the observed photoenhanced injection current.

In summary, we have presented an approach for separating injection from photogenerated currents in OSCs revealing the presence of non-negligible injection currents which are incident intensity dependent. By altering the nature of the Schottky barrier we have shown that these currents have their origin in photoenhanced charge injection at the PEDOT electrode. These results are likely to have significant implications for future measurements of the photogenerated currents that are observed in OSCs.

${ }^{1}$ C. J. Brabec, N. S. Sariciftci, and J. C. Hummelen, Adv. Funct. Mater. 11, 15 (2001).

${ }^{2}$ H. Frohne, D. C. Müller, and K. Meerholz, ChemPhysChem 3, 707 (2002).

${ }^{3}$ H. Frohne, S. E. Shaheen, C. J. Brabec, D. C. Müller, N. Serdar Sariciftci, and K. Meerholz, ChemPhysChem 3, 795 (2002).

${ }^{4}$ X. Wei, M. Raikh, Z. V. Vardeny, Y. Yang, and D. Moses, Phys. Rev. B 49, 17480 (1994).

${ }^{5}$ H. Neugebauer, C. Kvarnström, A. Cravino, T. Yohannes, and N. S. Sariciftci, Synth. Met. 116, 115 (2001).

${ }^{6} \mathrm{D}$. Long, Optical and Infrared Detectors (Topics in Applied Physics, Vol. 19, 2nd ed., edited by R. J. Keyes (Springer, Berlin, 1980), pp 101-147.

${ }^{7}$ J. S. Sandhu, A. P. Heberle, B. W. Alphenaar, and J. R. A. Cleaver, Appl. Phys. Lett. 76, 1507 (2000)

${ }^{8}$ E. W. McFarland and J. Tang, Nature (London) 421, 616 (2003).

${ }^{9}$ B. G. Streetman and S. Banerjee, Solid State Electronic Devices, 5th ed. (Prentice-Hall, Englewood Cliffs, NJ, 2000).

${ }^{10}$ K. Saito and S. Kobayashi, Jpn. J. Appl. Phys., Part 2 42, L781 (2003).

${ }^{11}$ S. E. Shaheen, C. J. Brabec, N. S. Sariciftci, F. Padinger, T. Fromherz, and J. C. Hummelen, Appl. Phys. Lett. 78, 841 (2001).

${ }^{12}$ J. Chappell, D. G. Lidzey, P. C. Jukes, A. M. Higgins, R. L. Thompson, S. O'Connor, I. Grizzi, R. Fletcher, J. O'Brien, M. Geoghegan, and R. A. L. Jones, Nat. Mater. 2, 616 (2003).

${ }^{13}$ J. K. J. van Duren, J. Loos, F. Morrissey, C. M. Leewis, K. P. H. Kivits, L. J. van IJzendoorn, M. T. Rispens, J. C. Hummelen, and R. A. J. Janssen, Adv. Funct. Mater. 12, 665 (2002). 